\title{
Double lepton pair production with electron capture in relativistic heavy-ion collisions
}

\author{
A. N. Artemyev ${ }^{1,2}$, V. G. Serbo ${ }^{3,4, a}$, A. Surzhykov ${ }^{1, b}$ \\ ${ }^{1}$ Helmholtz-Institut Jena, 07743 Jena, Germany \\ 2 St. Petersburg State Polytechnical University, St. Petersburg 195251, Russia \\ ${ }^{3}$ Sobolev Institute of Mathematics, 630090 Novosibirsk, Russia \\ ${ }^{4}$ Novosibirsk State University, 630090 Novosibirsk, Russia
}

Received: 19 February 2014 / Accepted: 18 March 2014 / Published online: 4 April 2014

(C) The Author(s) 2014. This article is published with open access at Springerlink.com

\begin{abstract}
We present a theoretical study of a double leptonpair production in ultra-relativistic collision between two bare ions. Special emphasis is placed to processes in which creation of (at least one) $e^{+} e^{-}$pair is accompanied by the capture of an electron into a bound ionic state. To evaluate the probability and cross section of these processes we employ two approaches based on (i) the first-order perturbation theory and multipole expansion of Dirac wavefunctions, and (ii) the equivalent photon approximation. With the help of such approaches, detailed calculations are made for the creation of two bound-free $e^{+} e^{-}$pairs as well as of bound-free $e^{+} e^{-}$ and free-free $\mu^{+} \mu^{-}$pairs in collisions of bare lead ions, $\mathrm{Pb}^{82+}$. The results of the calculations indicate that observation of the double lepton processes may become feasible at the LHC facility.
\end{abstract}

\section{Introduction}

Strong electromagnetic fields, induced in relativistic ionion as well as ion-atom collisions, may lead to a creation of electron-positron pairs. A large number of experimental and theoretical works have been performed to explore this quantum electrodynamic (QED) phenomenon [1-3]. However, while in the past most of the studies have dealt with single $e^{+} e^{-}$pairs, much of today interest is focused also on the multiple pair production. Such a process, in which few electrons and positrons are created in a single collision event, is of high order in the electromagnetic coupling $\alpha$ and is predicted to have a sufficiently large cross section $[4,5]$. Its analysis can help, therefore, to improve our understanding of the non-linear QED effects in quantum vacuum.

\footnotetext{
a e-mail: serbo@math.nsc.ru

b e-mail: a.surzhykov@gsi.de
}

Many attempts, based on coincidence observation of emitted electrons (or positrons), were made to detect the multiple production of free $e^{+} e^{-}$pairs. Due to a large background signal these measurements were unsuccessful, thus indicating a need for an alternative scenario of the experiment. This scenario can be provided by the investigation of multiple $e^{+} e^{-}$creation events accompanied by the electron capture into bound ionic states. Even though the bound-free pair production is much less probable than the free-free one, its experimental observation is feasible via detection of (one or few) down-charged ions. Such an experiment is likely to be performed at the LHC facility, whose very forward detectors allow efficient ion counting, and will reveal new information as regards the physics of extremely strong fields.

In order to support future LHC experiments, we present a theoretical study of a double lepton-pair production in ultrarelativistic collisions between bare nuclei. Especially, we focus on the creation of two bound-free $e^{+} e^{-}$pairs in which electrons are captured into bound states of either the same,

$Z_{1}+Z_{2} \rightarrow\left(Z_{1}+e^{-}+e^{-}\right)_{1 s^{2}}+Z_{2}+e^{+}+e^{+}$,
$Z_{1}+Z_{2} \rightarrow Z_{1}+e^{+}+e^{+}+\left(Z_{2}+e^{-}+e^{-}\right)_{1 s^{2}}$,

or two different ions,

$Z_{1}+Z_{2} \rightarrow\left(Z_{1}+e^{-}\right)_{1 s}+\left(Z_{2}+e^{-}\right)_{1 s}+e^{+}+e^{+}$.

Here we assume, moreover, the most probable case when the ground ionic states are populated in the course of the capture. The processes (1)-(2) and (3) can be investigated experimentally by detecting residual helium-like or two hydrogen-like ions, and without the need for observation of positrons. These measurements can become feasible in the near future owing to (relatively) large corresponding cross sections which, being proportional to $\alpha^{14}$, may reach the order of $10 \mathrm{mb}$. 
Beside the reactions (1)-(3), we consider-as the third scenario-the simultaneous production of a bound-free $e^{+} e^{-}$and a free-free $\mu^{+} \mu^{-}$pair:

$$
\begin{aligned}
& Z_{1}+Z_{2} \rightarrow\left(Z_{1}+e^{-}\right)_{1 s}+Z_{2}+\mu^{+}+\mu^{-}+e^{+} \\
& Z_{1}+Z_{2} \rightarrow Z_{1}+\mu^{+}+\mu^{-}+e^{+}+\left(Z_{2}+e^{-}\right)_{1 s} .
\end{aligned}
$$

Even though the experimental study of this process is rather cumbersome and requires coincidence measurement of a down-charged ion and emitted muon (or antimuon), it can be useful in the general discussion of electromagnetic processes at LHC. The theoretical background for the analysis of all three (double lepton) processes is discussed in Sect. 2. We show, in particular, that the computation of the cross sections can be traced back to the probability of pair production at a particular impact parameter. In the present work, two approaches were used to evaluate such a probability: apart from the first-order perturbation theory, we also employed analytical expressions derived from the equivalent photon approximation (EPA). Detailed calculations based on these two theories are presented in Sect. 3 for collisions of bare lead nuclei $\mathrm{Pb}^{82+}$ moving with Lorentz factors $\gamma=1,500$ and 3,000. For these typical LHC parameters, we predict up to tens of thousands double-pair production events per hour which can be measured by modern particle detectors. A brief summary of these results is given in Sect. 4 .

Relativistic units $c=\hbar=1$ and $\alpha \approx 1 / 137$ are used throughout the paper unless stated otherwise.

\section{Theoretical background}

In order to study the (double) lepton-pair production in ultrarelativistic heavy-ion collisions we employ here the impactparameter approach. Within such an approach, the projectile ion is assumed to move along a classical straight-line trajectory with an impact parameter $\rho$ and velocity $v$ as defined with respect to the target ion. The cross section of the pair creation is then given simply by

$\sigma=\int_{R_{1}+R_{2}}^{\infty} P(\rho) \mathrm{d}^{2} \rho=2 \pi \int_{R_{1}+R_{2}}^{\infty} P(\rho) \rho \mathrm{d} \rho$,

where $R_{1}+R_{2}$ is the sum of nuclear radii of colliding ions and the $P(\rho)$ is the probability of the process. As seen from this expression, the knowledge of the impact-parameter dependence of the $P(\rho)$ is crucial for the computation of the cross section $\sigma$. In the next sections we discuss, therefore, how the probability $P(\rho)$ can be evaluated for both the single and the double lepton-pair production.
2.1 Probability of a single $e^{+} e^{-}$pair production

\subsubsection{Equivalent photon approximation}

The creation of a single electron-positron pair in ion-ion collisions has been intensively studied over the last decades (see Ref. [3] and references therein). For ultra-relativistic energies, this process can be described by means of the equivalent photon approximation (EPA) which treats the electromagnetic field of a fast moving ion as a short pulse of linearly polarized light. The calculation of the $e^{+} e^{-}$cross section can be traced back, therefore, to the probability of pair photo-production. For example, if collision of two nuclei with charges $Z_{1}$ and $Z_{2}$ is accompanied by a creation of a free positron and an electron in the ground state of the "second" ion

$Z_{1}+Z_{2} \rightarrow Z_{1}+e^{+}+\left(Z_{2}+e^{-}\right)_{1 s}$,

the cross section of this process can be written

$\mathrm{d} \sigma_{e e}\left(Z_{2}, Z_{1}\right)=\mathrm{d} n_{\gamma}\left(\omega_{\mathrm{L}}, \rho\right) \sigma_{\gamma}\left(\omega_{\mathrm{L}}\right)$.

Here, $\mathrm{d} n_{\gamma}\left(\omega_{\mathrm{L}}, \rho\right)$ is the number of (virtual) photons with energy $\omega_{\mathrm{L}}$, produced by the "first" nucleus and seen in the rest frame of the "second" nucleus. The $\sigma_{\gamma}\left(\omega_{\mathrm{L}}\right)$ denotes then the cross section for the bound-free pair production following impact of the photon with energy $\omega_{\mathrm{L}}$ on the "second" nucleus, $\omega_{\mathrm{L}}+Z_{2} \rightarrow e^{+}+\left(Z_{2}+e^{-}\right)_{1 s}$.

The evaluation of the cross section $\sigma_{\gamma}\left(\omega_{\mathrm{L}}\right)$ and the photon number $\mathrm{d} n_{\gamma}$ has been discussed previously in Refs. [5, 6]. In the present study, therefore, we will restrict ourselves to a short account of the basic formulas and ideas, which are needed to derive the $\sigma_{e e}$ and probability $P(\rho)$ of the boundfree pair production. Within the framework of the EPA and the leading logarithmic approximation, the number of equivalent photons for large impact parameters, $1 / m \ll \rho \ll$ $\gamma_{\mathrm{L}} / \omega_{\mathrm{L}}$, reads [5]

$\mathrm{d} n_{\gamma}\left(\omega_{\mathrm{L}}, \rho\right)=\frac{Z_{1}^{2} \alpha}{\pi^{2}} \frac{\mathrm{d} \omega_{\mathrm{L}}}{\omega_{\mathrm{L}}} \frac{\mathrm{d}^{2} \rho}{\rho^{2}}$,

where $\gamma_{\mathrm{L}}$ is a Lorentz factor of a "first" nucleus in the rest frame of the "second" one. Inserting this expression into Eq. (8), we can find the cross section of the bound-free pair production:

$$
\begin{aligned}
\mathrm{d} \sigma_{e e}\left(Z_{2}, Z_{1}\right) & =\frac{Z_{1}^{2} \alpha}{\pi^{2}} \frac{\mathrm{d}^{2} \rho}{\rho^{2}} \int_{2 m}^{\infty} \frac{\mathrm{d} \omega_{\mathrm{L}}}{\omega_{\mathrm{L}}} \sigma_{\gamma}\left(\omega_{\mathrm{L}}\right) \\
& =\frac{Z_{1}^{2} \alpha}{\pi^{2}} \frac{\mathrm{d}^{2} \rho}{\rho^{2}} \frac{274}{315} \frac{\alpha\left(Z_{2} \alpha\right)^{5}}{m^{2}} f\left(Z_{2}\right)
\end{aligned}
$$


Here, the integration over the photon energy $\omega_{\mathrm{L}}$ employs the explicit form of the cross section $\sigma_{\gamma}\left(\omega_{\mathrm{L}}\right)$ as obtained within the well-known Sauter approximation (see e.g. Eq. (44) in Ref. [6]). The factor $f\left(Z_{2}\right)$, moreover, accounts for the difference between the Sauter predictions and rigorous relativistic calculations of the $e^{+} e^{-}$photo-production [7] and is inserted into Eq. (10) to improve the accuracy of calculations. For the collisions of bare lead ions, which will be discussed in Sect. 3, this factor is $f(82)=0.216$.

By comparing the cross section (10) with Eq. (6) one immediately derives the probability of a single bound-free $e^{+} e^{-}$pair production:

$P_{e e}\left(\rho ; Z_{2}, Z_{1}\right)=\frac{A\left(Z_{2}, Z_{1}\right)}{(m \rho)^{2}}, \quad \frac{1}{m} \ll \rho \ll \frac{\gamma_{\mathrm{L}}}{\omega_{\mathrm{L}}}$,

where the coefficient $A$ depends only on the charges of colliding nuclei and is given by

$A\left(Z_{2}, Z_{1}\right)=\left(Z_{1} \alpha\right)^{2}\left(Z_{2} \alpha\right)^{5} f\left(Z_{2}\right) \frac{274}{315 \pi}$.

These expressions describe the process (7) in which the electron is captured into the ground state of the "second" ion. Of course, the Eqs. (11)-(12) can also be applied to calculate the probability of the creation of an electron bound to the "first" nucleus; this would require just a substitution of charges $Z_{1}$ and $Z_{2}$.

Equation (11) is valid for large values of $\rho \gg 1 / m$, which give the main logarithmic contribution to the single pairproduction cross section $d \sigma_{e e}$. In contrast, if few $e^{+} e^{-}$pairs are created in course of ion-ion collision, the region of small impact parameters, $R_{1}+R_{2}<\rho \lesssim 1 / m$, plays a significant role, as will be shown in the next section. Since Eq. (11) is not justified in such a region, another approximation of $P_{e e}$ needs to be used. Based on the results of fully relativistic perturbative calculations $[8,9]$, which predict that the growth of the pair-production probability with decreasing $\rho$ slows down and eventually stops at a distance of a few Compton wavelengths, we conjecture that

$$
P_{e e}\left(\rho ; Z_{2}, Z_{1}\right) \approx P_{e e}\left(1 / m ; Z_{2}, Z_{1}\right)=A\left(Z_{2}, Z_{1}\right),
$$

for $R_{1}+R_{2}<\rho \lesssim 1 / \mathrm{m}$. The validity of such a naïve assumption will be discussed in Sect. 3 where we show that calculations based on Eq. (13) can reproduce the $e^{+} e^{-}$production cross sections with an accuracy of about $20 \%$.

\subsubsection{First-order perturbation theory}

Beside the equivalent photon approximation, which will be used here for rough estimations, one can employ the firstorder perturbation theory and relativistic Dirac wavefunctions in order to calculate the probability $P_{e e}$. Within such an approach, the transition amplitude $a_{e e}(\rho)=i \gamma Z_{1} e^{2} \int \mathrm{d} t \mathrm{e}^{i \omega t} \int \mathrm{d} \boldsymbol{r} \psi_{e^{-}}^{\dagger}(\boldsymbol{r}) \frac{1-v \alpha_{3}}{r^{\prime}} \psi_{e^{+}}(\boldsymbol{r})$

is a "building block" from which all the properties of the bound-free pair-production process (7) can be calculated [8,9]. In this expression, $\omega=E_{e^{+}}+E_{e^{-}}$is the sum of the total energies of emitted positron and bound electron, and $\alpha_{3}=\alpha_{z}$ is the Dirac matrix. Moreover, the $e^{+} e^{-}$pair production happens because of the Liénard-Wiechert potential $\left(1-v \alpha_{3}\right) / r^{\prime}$ of the "first" nucleus as seen in the rest frame of the "second" nucleus, which depends on the time-dependent distance $r^{\prime}=\sqrt{\left(x-\rho_{x}\right)^{2}+\left(y-\rho_{y}\right)^{2}+\gamma^{2}(z-v t)^{2}}$.

The computation of the transition amplitude (14) is significantly simplified if the standard radial-angular representation of the bound electron $\psi_{e^{-}}(\boldsymbol{r})$ and positron $\psi_{e^{+}}(\boldsymbol{r})$ functions is applied. For the continuum positron wave this representation can be achieved upon expansion of the $\psi_{e^{+}}(\boldsymbol{r})$ into multipole (partial) components, characterized by a welldefined parity and total angular momentum. The partial-wave analysis is routine and has been performed in a large number of studies of various atomic processes [8-12]. In the present work, we used about 40 partial waves to calculate the amplitude $a_{e e}(\rho)$ and then the bound-free $e^{+} e^{-}$pair-production probability:

$P_{e e}\left(\rho ; Z_{2}, Z_{1}\right)=\int \mathrm{d} \boldsymbol{p}_{+}\left|a_{e e}(\rho)\right|^{2}$,

where the integration runs over the momentum $\boldsymbol{p}_{+}$of emitted positron and the proper summation over the particle spins is implied (see Refs. $[9,10]$ for further details).

\subsection{Probability of a double $e^{+} e^{-}$pair production}

Based on the probability $P_{e}$, derived for the process (7), one can investigate also the double bound-free pair production in a single collision between two nuclei. Two scenarios have to be considered for such a collision. In the first one, both electrons are captured by the same nucleus, cf. Eqs. (1) and (2). The probability of such a process can be expressed as a product of probabilities of a single pair production accompanied by the formation of (i) a hydrogen-like and, as a second step, (ii) a helium-like ion. If the electrons are created in the ground state of the ion with a nuclear charge $Z_{2}$, this probability reads

$P_{2 e e}\left(\rho ; Z_{2}, Z_{1}\right)=\frac{1}{2} P_{e e}\left(\rho ; Z_{2}, Z_{1}\right) P_{e e}\left(\rho ; Z_{2}, Z_{1}\right)$.

Here, we neglected the interaction between bound electrons and just introduced the factor $1 / 2$ to account for the Pauli exclusion principle. Such an independent-particle model is usually well justified for atomic processes with heavy ions 
and has been successfully employed in a large number of studies $[13,14]$.

By inserting the probability $P_{2 e e}$ into Eq. (6) one can derive the cross section

$$
\begin{aligned}
\sigma_{2 e e}\left(Z_{2}, Z_{1}\right) & =\int_{R_{1}+R_{2}}^{\infty} P_{2 e e}\left(\rho ; Z_{2}, Z_{1}\right) \mathrm{d}^{2} \rho \\
& =\frac{1}{2} \int_{R_{1}+R_{2}}^{\infty}\left[P_{e e}\left(\rho ; Z_{2}, Z_{1}\right)\right]^{2} 2 \pi \rho \mathrm{d} \rho
\end{aligned}
$$

of the process (1). While the evaluation of this cross section is the topic of Sect. 3, here we just note that the main contribution to the integral in Eq. (17) is due to the region $\rho \lesssim 1 / m$ since at $\rho \gg 1 / m$ the integrand $\left[P_{e e}\left(\rho ; Z_{2}, Z_{1}\right)\right]^{2} \rho \propto 1 / \rho^{3}$ drops rapidly to zero. This observation confirms the importance of small impact parameters $\rho$ for the analysis of double pair-production processes.

In the second scenario, the ultra-relativistic collision between two nuclei leads to a formation of two hydrogenlike ions in the process (3), whose probability can be written

$$
\begin{aligned}
& P_{e e+e e}\left(\rho ; Z_{1}, Z_{2}\right)=\frac{1}{2}\left[P_{e e}\left(\rho ; Z_{2}, Z_{1}\right) P_{e e}\left(\rho ; Z_{1}, Z_{2}-1\right)\right. \\
& \left.\quad+P_{e e}\left(\rho ; Z_{1}, Z_{2}\right) P_{e e}\left(\rho ; Z_{2}, Z_{1}-1\right)\right] .
\end{aligned}
$$

Again, here we treat the double-pair production as a two-step process. For instance, the first line of Eq. (16) represents the probability that (i) the virtual photon emitted by the nucleus $Z_{1}$ creates a bound electron and a positron in the field of bare ion with the charge $Z_{2}$, and (ii) the production of the second $e^{+} e^{-}$pair in the field of the "first" nucleus is induced by an impact with an electromagnetic field of the hydrogen-like ion $\left(Z_{2}+e^{-}\right)_{1 s}$. The factor $1 / 2$ accounts, moreover, for the identity of final states, as required by the Poisson statistics.

In order to further simplify Eq. (18) we recall that small impact parameters, $\rho \lesssim 1 / m$, provide the main contribution to the double-pair production cross sections. In this parameter range the nucleus of the hydrogen-like ion $(Z-1)_{1 s}$ is weakly screened by the (bound) electron cloud with the mean square radius $\sqrt{\left\langle r^{2}\right\rangle}=\sqrt{3} /[(Z-1) \alpha m]>1 / m$. The electromagnetic field, produced by such an ion and as "seen" at $\rho \lesssim 1 / m$ is very similar, therefore, to that of the bare nucleus $Z$. This allows us to approximate $P_{e e}\left(\rho ; Z_{1,2}, Z_{2,1}-1\right) \approx$ $P_{e e}\left(\rho ; Z_{1,2}, Z_{2,1}\right)$ and to re-write Eq. (18) as

$$
P_{e e+e e}\left(\rho ; Z_{1}, Z_{2}\right) \approx P_{e e}\left(\rho ; Z_{2}, Z_{1}\right) P_{e e}\left(\rho ; Z_{1}, Z_{2}\right)
$$

In Sect. 3 we will make use of this probability to investigate the cross section of the pair-production process (3).
2.3 Probability of a free $\mu^{+} \mu^{-}$and bound-free $e^{+} e^{-}$pair production

Up to the present, we have applied the $P_{e e}$ to evaluate probabilities of various electron-positron production processes. The ultra-relativistic heavy-ion collisions may lead also to the creation of other leptons. Of special interest for the LHC physics, for example, are muon-antimuon pairs. While the production of single and double free-free $\mu^{+} \mu^{-}$pairs was considered in Ref. [15], here we study the process (4)-(5), in which free-free $\mu^{+} \mu^{-}$and bound-free $e^{+} e^{-}$pairs are created. If the electron is captured into a bound state of the "second" nucleus, the probability of such a process is

$P_{e e+\mu \mu}(\rho)=P_{\mu \mu}(\rho) P_{e e}\left(\rho ; Z_{2}, Z_{1}\right)$,

where $P_{\mu \mu}(\rho)$ is given, within the leading logarithmic approximation, by [15]:

$P_{\mu \mu}(\rho)=\frac{28}{9 \pi^{2}} \frac{\left(Z_{1} \alpha Z_{2} \alpha\right)^{2}}{(\mu \rho)^{2}} \Phi(\rho, \gamma)$.

In this expression, $\gamma$ is the Lorentz factor of the nuclei in the laboratory (collider) frame, $\mu$ is the muon mass, and the function $\Phi(\rho, \gamma)$ reads

$\Phi(\rho, \gamma)=\left(4 \ln \frac{\gamma}{\mu \rho}+\ln \frac{\rho}{R}\right) \ln \frac{\rho}{R}$

for $R \ll \rho \leq \gamma / \mu$, and

$\Phi(\rho, \gamma)=\left(\ln \frac{\gamma^{2}}{\mu^{2} \rho R}\right)^{2}$

for $\gamma / \mu \leq \rho \ll \gamma^{2} /\left(\mu^{2} R\right)$. In the past, the approximate formulas (21)-(23) were successfully employed to study muonantimuon pair production in heavy-ion collisions. For the $\mathrm{Pb}-$ $\mathrm{Pb}$ scattering, for example, their predictions were found to be in a $10-15 \%$ agreement with the results of rigorous relativistic calculations [16]. Below, we will use the $P_{\mu \mu}(\rho)$ together with Eq. (11) and (20) in order to calculate the cross section of the free $\mu^{+} \mu^{-}$and bound-free $e^{+} e^{-}$pair production.

\section{Results and discussion}

As discussed in the previous section, the probabilities and, hence, the cross sections of the double lepton-pair production processes (1)-(5) can be expressed in terms of the (singlepair) probability $P_{e e}$. Beside the standard first-order perturbative approach, we have also derived the analytical expressions from the equivalent photon approximation to find the $P_{e e}$. Before employing these EPA formulas for the estimation 


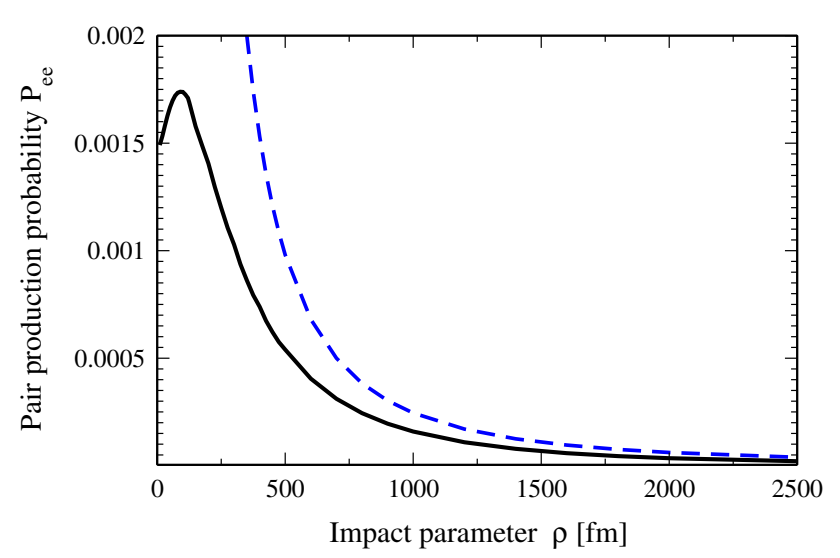

Fig. 1 The probability $P_{e e}$ of a single $e^{+} e^{-}$pair production in the collision of two led nuclei $\mathrm{Pb}^{82+}$ accompanied by the electron capture into the ground ionic state. Results of the perturbative relativistic calculations (solid line) are compared with the predictions of Eq. (11), displayed by the dashed line. Calculations were performed for various impact parameters $\rho$ and ion energy $T_{p}=100 \mathrm{GeV} / \mathrm{u}$

of the pair-creation cross sections, let us briefly discuss their validity both for small and large impact parameters $\rho$. In order to examine the performance of Eq. (11), used to describe the probability $P_{e e}$ for $\rho \gg 1 / m$, we compared its predictions with those of the perturbation theory (14)-(15). Perturbative calculations have been carried out for the collision of two lead nuclei $\mathrm{Pb}^{82+}$ moving with the energy $T_{p}=100 \mathrm{GeV} / \mathrm{u}$ in the laboratory frame. We restricted our analysis to thisrelatively low-energy since the probability (15) is known to remain almost unchanged for $T_{p} \gtrsim 100 \mathrm{MeV} / \mathrm{u}$ [9] and, moreover, a good convergence of the partial-wave expansion of the positron wavefunction can be achieved in such an (energy) region. As seen from Fig. 1, the perturbative results and approximation (11) show better agreement as the impact parameter $\rho$ increases. For instance, while for $\rho=386 \mathrm{fm}$, which corresponds to the electron Compton wavelength $1 / \mathrm{m}$, Eq. (11) overestimates the results of rigorous relativistic calculations by more than factor of two, the discrepancy between the results is about $40 \%$ for $\rho=2,000 \mathrm{fm}$. Such an accuracy is sufficient for the estimation of the pair-production cross sections as can be observed at the LHC facility.

If for the large impact parameters, $\rho \gg 1 / m$, the probability $P_{e e}$ of a single electron-positron pair production is approximated - within the EPA - by Eq. (11), in the region $2 R<\rho \lesssim 1 / m$ we use the naïve approach $P_{e e} \approx A$ with $A$ given by Eq. (12). For the ultra-relativistic collision between two lead nuclei the coefficient $A$ is as large as

$$
\begin{aligned}
A \equiv A(Z=82, Z=82) & =\frac{274 \alpha^{7}}{315 \pi}(82)^{7} f(Z=82) \\
& =1.65 \cdot 10^{-3}
\end{aligned}
$$

which is in reasonable agreement with the results of the relativistic partial-wave theory obtained in the range $\rho \lesssim 400 \mathrm{fm}$ (cf. solid line in Fig. 1). Moreover, the approximation $P_{e e} \approx$ $A$ can also be applied to estimate the cross section of the $e^{+} e^{-}$pair production in the vicinity of nucleus. For instance, by performing integration in Eq. (6) from $\rho=1 / 2 m$ to $\rho=1 / m$ we find

$\sigma_{e e}(\Delta \rho)=\int_{1 / 2 m}^{1 / m} P_{e e}(\rho) \mathrm{d}^{2} \rho=\frac{3 \pi}{4 m^{2}} A=5.8$ barn

for the $\mathrm{Pb}^{82+}-\mathrm{Pb}^{82+}$ case. Comparison of this prediction with the result $\sigma_{e e}(\Delta \rho) \approx 4$ barn, obtained for the same range of impact parameters and within the non-perturbative coupled-channel theory [17], again justifies the use of Eq. (13).

Having proved the validity of Eqs. (11) and (13) we are ready now to employ them-along with the perturbative theory-for the computation of the cross sections of double lepton-pair production. Similar to before, we focus on the ultra-relativistic $\mathrm{Pb}^{82+}-\mathrm{Pb}^{82+}$ collisions and will study them for the scenario when two beams with Lorentz factors $\gamma_{1}=\gamma_{2} \equiv \gamma=1,500$ and 3,000, as defined in the collider (center-of-mass) system, and the luminosity $\mathcal{L}=10^{27} \mathrm{~s}^{-1} \mathrm{~cm}^{-2}$ move toward each other. As discussed already in Sect. 2.2, two $e^{+} e^{-}$pairs can be created in these collisions accompanied by the capture of the electrons either (i) by the same (1)-(2) or (ii) by two different (3) ions. By using Eqs. (6), (16) and (19), we easily find that the cross sections of these two processes are related to each other by

$\sigma_{e e+e e}=2 \sigma_{2 e e}=\int_{2 R}^{\infty}\left[P_{e e}(\rho, Z, Z)\right]^{2} 2 \pi \rho \mathrm{d} \rho$,

where $R_{1}=R_{2}=R$ is the radius of the lead nucleus. For the further evaluation of this expression, the knowledge about the $P_{e e}$ is needed. If, for example, the results of relativistic partial-wave calculations (cf. solid line in Fig. 1) are employed in Eq. (26), one finds, upon numerical integration over the impact parameter,

$\sigma_{e e+e e}=2 \sigma_{2 e e}=11 \mathrm{mb}$.

This result can be compared also with the prediction based on the approximations (11) and (13). Namely, since the $\left[P_{e e}(\rho)\right]^{2}$ scales as $\sim 1 / \rho^{4}$ for the large impact parameters (see Eq. (11) and Fig. 1), the main contribution to the integral on the right-hand side of (26) originates from the region $\rho \lesssim 1 / m$ where Eq. (13) is conjectured to describe the $e^{+} e^{-}$production probability. If we employ this simple model in Eq. (26), we obtain 


$$
\sigma_{e e+e e}=2 \sigma_{2 e e} \cong \int_{2 R}^{1 / m} A^{2} 2 \pi \rho \mathrm{d} \rho=\frac{\pi A^{2}}{m^{2}}=12.6 \mathrm{mb}
$$

which is in reasonable agreement with the numerical relativistic result (27). Our estimates of the cross sections $\sigma_{2 e e}$ and $\sigma_{e e+e e}$ suggest that about 40,000 double pair-production events (3) per hour may occur in course of $\mathrm{Pb}^{82+}-\mathrm{Pb}^{82+}$ ultra-relativistic collisions at the LHC. Moreover, one can observe approximately the same number of $e^{+} e^{-}$events in which the helium-like ions will be formed in either of colliding beams, i.e. when the process (1) or (2) will take place.

As mentioned already above, the bound-free $e^{+} e^{-}$pair might be also created together with the free muon and antimuon, cf. Eqs. (4) and (5). The cross section of such a-rather exotic-process,

$$
\begin{aligned}
\sigma_{e e+\mu \mu}(\gamma) & =\int_{2 R}^{\infty} P_{e e+\mu \mu}(\rho ; Z, Z) 2 \pi \rho \mathrm{d} \rho \\
& =\int_{2 R}^{\infty} P_{\mu \mu}(\rho ; Z, Z, \gamma) P_{e e}(\rho ; Z, Z) 2 \pi \rho \mathrm{d} \rho,
\end{aligned}
$$

can be derived from Eqs. (6) and (20). In contrast to $\sigma_{2 e e}$ and $\sigma_{e e+e e}$ this cross section exhibits an evident dependence on the Lorentz factor $\gamma$ which arises from the $\mu^{+} \mu^{-}$production probability (21)-(23). In order to compute the $\sigma_{e e+\mu \mu}$, therefore, one has to specify the energy of colliding ions. For example, by choosing the values $\gamma=1,500$ and 3,000, typical for $\mathrm{LHC} \mathrm{Pb}^{82+}-\mathrm{Pb}^{82+}$ experiments, we find

$\sigma_{e e+\mu \mu}(1,500)=2.2 \mathrm{mb}, \quad \sigma_{e e+\mu \mu}(3,000)=2.6 \mathrm{mb}$,

which is comparable with the cross sections of the double free-free $\mu^{+} \mu^{-}$pair production, $\sigma_{2 \mu \mu} \sim 1 \mathrm{mb}$ [15]. The results (30) are based on the calculations of the $e^{+} e^{-}$production probability, performed within the framework of the perturbation theory (14)-(15). If, in contrast, the probability $P_{e e}$ is approximated by Eqs. (11) and (13), one can derive for $Z_{1}=Z_{2}=Z=82$ :

$$
\begin{aligned}
\sigma_{e e+\mu \mu}(\gamma) & \sim \int_{2 R}^{1 / m} A P_{\mu \mu}(\rho ; Z, Z, \gamma) 2 \pi \rho \mathrm{d} \rho \\
& =6 A \frac{28}{27 \pi} \frac{(\alpha Z)^{4}}{\mu^{2}} \int_{2 R}^{1 / m} \Phi(\rho, \gamma) \frac{\mathrm{d} \rho}{\rho} \\
& =1.8 \mathrm{mb}(\text { for } \gamma=1,500) \\
& =2.1 \mathrm{mb}(\text { for } \gamma=3,000) .
\end{aligned}
$$

Again, both results are in good agreement and imply that about 15,000 events (4) and (5) per hour can take place at the LHC collider. The detection of these events would require, however, a coincidence measurement the downcharged, hydrogen-like ions and emitted $\mu^{-}\left(\right.$or $\left.\mu^{+}\right)$. Due to the limited detector geometry, however, not all created muons can be registered and this might lead to a considerable reduction of the count rate.

The calculations discussed above have been made under the assumption that an electron is captured into the ground ionic state of either hydrogen- or helium-like heavy ions. The multiple $e^{+} e^{-}$pair production can also be accompanied by the formation of residual ions in their excited states. Based on the previous studies $[6,18]$, we estimate that the contribution of such a excited-state recombination to the total cross sections $\sigma_{2 e e}, \sigma_{e e+e e}$ and $\sigma_{e e+\mu \mu}$ does not exceed $25 \%$, which is well within the accuracy margin of the present calculations.

\section{Summary}

In summary, a theoretical study of double lepton-pair production with an electron capture in ultra-relativistic heavyion collisions has been presented. Special emphasis was given to the processes involving creation of (one or two) bound-free $e^{+} e^{-}$pairs. In order to estimate the probabilities of these processes at different impact parameters we used two independent approaches. The first one is traced back to the first-order perturbation theory and the partialwave expansion of the Dirac wavefunctions, while the second employs simple analytical expressions derived within the framework of the equivalent photon approximation. Based on these approaches, calculations have been performed for the typical LHC scenario in which two bare led ions $\mathrm{Pb}^{82+}$, moving with the Lorentz factor $\gamma=1,500$ and 3,000, collide with each other. For such a collision, we analyzed the probabilities and then the cross sections of the double $e^{+} e^{-}$ pair production accompanied by the formation of (i) a single helium-like (1)-(2) or (ii) two hydrogen-like ions (3), as well as (iii) the simultaneous creation of bound-free $e^{+} e^{-}$ and free-free $\mu^{+} \mu^{-}$pairs (4)-(5). The predictions of the relativistic partial-wave theory and the EPA approach were found to be in good agreement for all three processes, and they have indicated that up to tens of thousands of (pair production) events per hour can happen in the course of high- $\gamma$ collisions at the LHC facility. With such a remarkable event rate the experimental studies of the above processes are likely to become feasible in the near future and will reveal new and unique information on the quantum electrodynamics in extremely strong electromagnetic fields.

Acknowledgments We are grateful to R. Schicker who directed our attention to this problem and explained us the details of the ALICE 
experiment. The stimulating discussions with A. Milstein and A. Voitkiv are also highly acknowledged. The work is supported by the ExtreMe Matter Institute (EMMI). A. A. and A. S. acknowledge support from the Helmholtz Gemeinschaft (Nachwuchsgruppe VH-NG-421). V. G. S. is supported by the Russian Foundation for Basic Research under the grant 13-02-00695.

Open Access This article is distributed under the terms of the Creative Commons Attribution License which permits any use, distribution, and reproduction in any medium, provided the original author(s) and the source are credited.

Funded by $\mathrm{SCOAP}^{3}$ / License Version CC BY 4.0.

\section{References}

1. A. Belkacem, H. Gould, B. Feinberg, R. Bossingham, W.E. Meyerhof, Phys. Rev. Lett. 71, 1514 (1993)

2. M.C. Güçlüa, J. Lib, A.S. Umarb, D.J. Ernstb, M.R. Strayer, Ann. Phys. 272, 7 (1999)

3. G. Baur, K. Hencken, D. Trautmann, Phys. Rep. 453, 1 (2007)

4. G. Baur, K. Hencken, D. Trautmann, S. Sadovsky, Y. Kharlov, Phys. Rep. 364, 359 (2002)

5. R.N. Lee, A.I. Milsyein, V.G. Serbo, Phys. Rev. A 65, 022102 (2002)
6. A.N. Artemyev, U.D. Jentschura, V.G. Serbo, A. Surzhykov, Eur. Phys. J. C 72, 1935 (2012)

7. C.K. Agger, A.H. Sørensen, Phys. Rev. A 55, 402 (1997)

8. S.R. Valluri, U. Becker, N. Grün, W. Scheid, J. Phys. B At. Mol. Phys. 17, 4359 (1984)

9. U. Becker, N. Grün, W. Scheid, J. Phys. B At. Mol. Phys. 20, 2075 (1987)

10. A. Surzhykov, S. Fritzsche, J. Phys. B At. Mol. Phys. 38, 2711 (2005)

11. B. Najjari, A.B. Voitkiv, A. Artemyev, A. Surzhykov, Phys. Rev. A 80, 012701 (2009)

12. J. Eichler, Th Stöhlker, Phys. Rep. 439, 1 (2007)

13. A. Surzhykov, U.D. Jentschura, T. Stöhlker, S. Fritzsche, Phys. Rev. A 73, 032716 (2006)

14. A. Surzhykov, U.D. Jentschura, T. Stöhlker, S. Fritzsche, Eur. Phys. J. D 46, 27 (2008)

15. K. Hencken, E.A. Kuraev, V.G. Serbo, Phys. Rev. C 75, 034903 (2007)

16. K. Hencken, D. Trautmann, G. Baur, Phys. Rev. A 51, 1874 (1995)

17. A.J. Baltz, M.J. Rhoades, J. Weneser, Phys. Rev. A 47, 3444 (1993)

18. R.N. Lee, A.I. Milstein, V.M. Strakhovenko, Phys. Rev. A 69, 022708 (2004) 